\title{
Cevipabulin Succinate
}

National Cancer Institute

\section{Source}

National Cancer Institute. Cevipabulin Succinate. NCI Thesaurus. Code C96746.

The succinate salt form of cevipabulin, a synthetic, water soluble tubulin-binding agent with potential antineoplastic activity. Cevipabulin binds at the vinca-binding site on tubulin, but seems to act more similar to taxane-site binding agents in that it enhances tubulin polymerization and does not induce tubulin depolymerization. This stabilizes tubulin and prevents microtubule disassembly. The disruption in microtubule dynamics may eventually inhibit cell division and reduce cellular growth. 